\title{
Optical characterization of TiAlN/TiAlON/SiO 2 absorber for solar selective applications
}

\author{
L. Rebouta ${ }^{\text {a,* }}$, A. Pitães ${ }^{\text {a }}$, M. Andritschky ${ }^{\text {a }}$, P. Capela ${ }^{\text {a }}$, M.F. Cerqueira ${ }^{\text {a }}$, A. Matilainen ${ }^{\text {b }}$, K. Pischow ${ }^{\text {b }}$ \\ a Centre of Physics, University of Minho, Azurém, 4800-058 Guimarães, Portugal \\ b Savo Solar Oy, Insinöörinkatu 7, 50100 Mikkeli, Finland
}

\section{A R T I C L E I N F O}

Available online 17 September 2011

\section{Keywords:}

TiAlN

TiAINO

Reactive magnetron sputtering

PECVD

Solar selective absorber

\begin{abstract}
A B S T R A C T
Characterization of a TiAIN/TiAlON/SiO ${ }_{2}$ tandem absorber is reported in this contribution. The first two layers were deposited by magnetron sputtering and the third layer was prepared by plasma enhanced chemical vapour deposition (PECVD). The optimization was performed by determining the optical constants of individual layers by first measuring spectral transmittance and reflectance of the individual layers. Subsequently the measuring spectra were fitted using the SCOUT software and dielectric function of each layer was determined. The three layer stack absorber on copper was then designed using those optical properties. The thickness of the individual layers was optimized until a solar absorptance of 95.5\% was obtained resulting in a total thickness of about $215 \mathrm{~nm}(65 \mathrm{~nm} / 51 \mathrm{~nm} / 100 \mathrm{~nm}$ for the individual layers, respectively). A thermal emittance of $5 \%$ for an absorber temperature of $100{ }^{\circ} \mathrm{C}$ was obtained by analyzing the measuring data from a FTIR spectrometer with integrating sphere. During continuous thermal annealing at $278{ }^{\circ} \mathrm{C}$ for $600 \mathrm{~h}$ the absorptance decreased by $0.4 \%$.
\end{abstract}

(c) 2011 Elsevier B.V. All rights reserved.

\section{Introduction}

In last decades a big effort has been done in developing simple and effective structures for solar selective applications [1-5]. The selective absorbers should have high solar absorptance (low spectral reflectance for wavelengths $(\lambda)$ below $2500 \mathrm{~nm}$ ) and low thermal emittance (high spectral reflectance for $\lambda>2500 \mathrm{~nm}$ ). Different solutions have been used to reach those requirements, namely optical interference coating [4,5], multilayer gradient coating [6,7] and cermets composite coating $[3,8]$. The optical performance of these solutions, selective absorption in the solar spectrum and reflection in the thermal spectrum, is strongly dependent of its design, the optical constants of the constituents, thicknesses of the different layers and surface roughness.

The increase of operating temperatures has required solutions with improved oxidation resistance, maintaining the optical performance. Some transition metal nitrides have shown high chemical stability, high degree of spectral selectivity and also have improved oxidation resistance $[9,10]$, namely TiN and TiAlN, which have been used in the development of spectrally selective solar absorber [4,5,9-11]. The use oxynitrides has some advantages, not only in logistic terms because allows the use of same targets, but also because the transition metal oxynitrides have good properties for these applications [12] and can be combined with the nitrides. In the optical interference design the usual way is to use an infrared reflecting

\footnotetext{
* Corresponding author. Tel.: + 351253510472; fax: + 351253510461 E-mail address: rebouta@fisica.uminho.pt (L. Rebouta).
}

substrate covered by an antireflection layer with small refractive index. $\mathrm{SiO}_{2}$ has an adequate refractive index for an anti-reflection (AR) layer for solar selective absorbing surface, and has good properties such as negligible extinction coefficient and high thermal stability.

Within the frame of this work, an optical stack TiAlN/TiAlNxOy/SiO was designed and deposited on copper. Firstly, using the SCOUT software [13] and the combined reflectance and transmittance measurements, the optical constants of the considered materials were calculated. Secondly, those optical constants were then used to design the 3 layered stack for a solar selective absorber.

\section{Experimental details}

\subsection{Sample preparation and characterization}

TiAlN and TiAlNxOy thin films were deposited by dc magnetron sputtering on glass and copper substrates, under an $\mathrm{Ar} / \mathrm{N}_{2} / \mathrm{O}_{2}$ atmosphere with a base pressure of $2 \times 10^{-4} \mathrm{~Pa}$, from a TiAl target (titanium/aluminium, 50/50 at.\%) of 4 inch diameter. Nitride films were prepared with a variation of the nitrogen gas flow from 1.5 to $3 \mathrm{sccm}$, while for oxynitride films a reactive gas mixture (nitrogen + oxygen with a 17:3 ratio) was used, which was varied from 3 to $5 \mathrm{sccm}$. Argon flow was kept constant at $60 \mathrm{sccm}$.

A current density of $6.4 \mathrm{~mA} . \mathrm{cm}^{-2}$ applied to the target and a bias voltage of $-60 \mathrm{~V}$, applied to the substrate holder, were used in all depositions, which took place without external substrate heating. The working pressure was $0.4 \mathrm{~Pa}$, with a target-to-substrate distance of $8 \mathrm{~cm}$. 
The $\mathrm{SiO}_{2}$ layer was deposited, in a second coating chamber, by Plasma Enhanced Chemical Vapor Deposition (PECVD) using a Inductively Coupled Plasma (ICP) source and Octamethylcyclotetrasiloxane (OMCTS), $\left(\left(\mathrm{CH}_{3}\right)_{2} \mathrm{SiO}-\right)_{4}$, as a precursor. During those depositions a rf power of $3000 \mathrm{~W}$, an oxygen flow of $750 \mathrm{sccm}$ and precursor flow of $15 \mathrm{~g} / \mathrm{h}$ were used. The total pressure during deposition was $0.7 \mathrm{~Pa}$. Before the deposition, the substrates (glass and copper foil) were ultrasonic cleaned in acetone during $15 \mathrm{~min}$ and sputter etched at an Ar pressure of $4 \mathrm{~Pa}$ with a negative voltage of $600 \mathrm{~V}$ during $15 \mathrm{~min}$.

The structural properties of the films were studied by X-ray diffraction (XRD) employing a Bruker AXS Discover D8 operating with $\mathrm{CuK} \alpha$ radiation. Due to the low thickness films (around $60 \mathrm{~nm}$ ) grazing angle diffraction measurements have been performed for an angle of incidence of $1^{\circ}$. Optical measurements, in transmittance and reflectance modes, were performed in the wavelength range of $250-2500 \mathrm{~nm}$, using a Shimadzu UV-3101PC spectrometer. The reflectance measurements were performed with an incidence angle of $8^{\circ}$ using an integrating sphere attachment of diameter $60 \mathrm{~mm}$ with circular beam entrance and sample port of $18 \mathrm{~mm}$. Al coated mirror was used as the reference mirror. A Fourier Transform Infrared (FTIR) spectrophotometer was used to cover the infrared wavelength range, $2.5-16 \mu \mathrm{m}$, which is equipped with an integrating sphere. The emittance was also measured with an emissometer AE-AD3 from Devices \& Service Company.

Normal thermal emittance is a weighted fraction between emitted radiation and the Planck black body distribution at $100{ }^{\circ} \mathrm{C}$ [15] and the normal solar absorptance, $\alpha_{\text {sol }}$, is defined as a weighted fraction between absorbed radiation and incoming solar radiation [15]. The solar spectrum, used here is defined according to the ASTM G173-03 Reference Spectra. The accelerated life-time testing were performed accordingly the principles presented in ref [14].

\subsection{Dielectric function model}

A theoretical analysis of transmittance and reflectance of films can be used to obtain the optical constants [16]. The used model characterizes the optical properties by a quantity called complex dielectric function [17], $\tilde{\varepsilon}_{r}=\varepsilon_{1}+i \varepsilon_{2}$, which is related with complex refractive index, $\tilde{n}^{2}=\tilde{\varepsilon}_{r}$. The complex refractive index is usually defined through the relation, $\tilde{n}=n+i \kappa$, where $n$ is the normal refractive index and $\kappa$ is the extinction coefficient, which is directly related with the absorption coefficient of the medium. These relations show that the complex refractive index and dielectric function are not independent, and from $\varepsilon_{1}$ and $\varepsilon_{2}$ it is possible to calculate $n$ and $\kappa$, using the Kramers-Kronig relation, and vice versa.

The experimental transmittance and reflectance curves were modelled by a commercial optical simulation program (SCOUT) [13] and the thickness and dielectric function of the different layers were calculated. Previously to the calculation of the optical properties of the films, a similar procedure was used to the glass and copper substrates. The obtained dielectric functions of the substrates were used in the remaining calculations.

The dielectric function of TiAlN, TiAlNxOy and $\mathrm{SiO}_{2}$ was considered as a contribution of intraband and interband transitions. The dielectric function was treated as the sum of Drude free carrier absorption, a number of bound harmonic oscillators, a term (OJL) proposed by O'Leary, Johnson and Lim [18], and a background:

$\tilde{\varepsilon} r=\tilde{\varepsilon}_{\text {background }}+\tilde{\varepsilon}_{\text {Drude }}+\sum \tilde{\varepsilon}_{\text {Lorentz }}+\tilde{\varepsilon}_{\text {OJL }}$

The Drude model (only used for TiAlN films), which represents unbound electron oscillators, describes the intraband transitions of the electrons in the conduction band. Bound harmonic oscillators [19] model was used to describe the interband transitions into the upper half of the conduction band. The OJL term follows the O'Leary model [18] and was used to describe the band gap transitions for which parabolic bands are assumed with tail states exponentially decaying into band gap. Details about the model and this adaptation can be found in the literature [20,21]. The parameters used in the fit are the optical band gap energy (Eg), the decay energy, to describe the exponential tail extending into the band gap, and the strength of the transition.

\section{Results and discussion}

The structure (crystallinity) of the samples was investigated by grazing incidence X-ray diffraction analysis and Raman scattering. Fig. 1a) shows the typical XRD spectra for TiAlN and TiAlOxNy films grown on glass substrates. These films have thicknesses around $60 \mathrm{~nm}$. The nitride films show some crystallinity and the diffraction peaks can be indexed with solid solution $(\mathrm{Ti}, \mathrm{Al}) \mathrm{N}$ of the cubic $\mathrm{NaCl}$ type structure. The patterns from oxynitrides indicated that they are amorphous. Fig. 1b) shows the typical Raman spectra for the same samples. It is clearly seen the presence of acoustic mode of TiAlN at approx. $250 \mathrm{~cm}^{-1}$ (related with vibrations of Ti ion) and the optic mode of TiN at $566 \mathrm{~cm}^{-1}$ (related with vibrations of $\mathrm{N}$ ion). Based on Raman study, the phases $\mathrm{TiO}_{2}$, $\mathrm{AlO}$ and AlN were not detected in the analyzed films. Comparing the different produced samples we verified that, increasing the $\mathrm{N}$ content, the optic mode (at $560 \mathrm{~cm}^{-1}$ ) decreases in intensity whereas the acoustic mode $\left(250 \mathrm{~cm}^{-1}\right)$ appears.

The optical spectroscopy in both transmittance and reflectance modes was used to characterize the optical properties of the
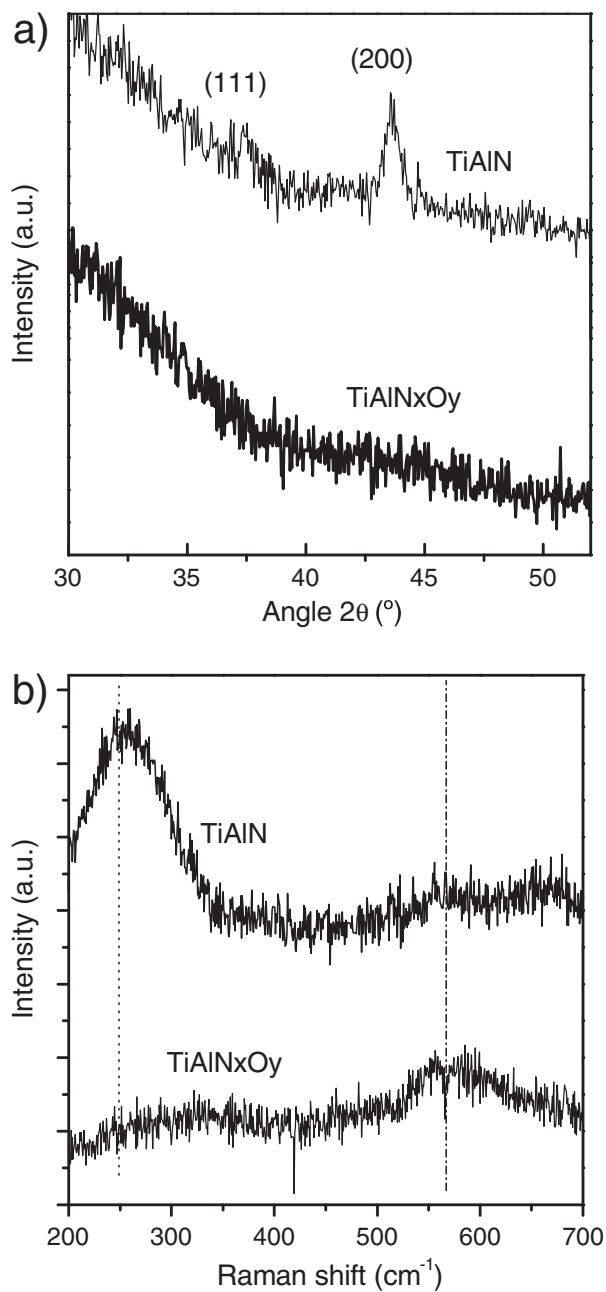

Fig. 1. a) X-Ray patterns of representative nitride and oxynitride layers deposited on glass substrates; and b) typical Raman spectra for the same samples. 
films. Fig. 2 depicts the optical transmittance and reflectance data for nitride samples prepared with different $\mathrm{N}_{2}$ flows (Fig. 2a) and oxynitride samples prepared with different gas mixture flows (Fig. 2b), as indicated in the figures. The nitrides have higher reflectance while the oxynitrides have higher transmittance. This is in accordance with the metallic behaviour of these nitrides and the semiconducting properties of these type of oxynitrides [22]. The different reactive gases flows allowed obtaining a wide range of reflectance and transmittance values, from which it is possible to select the layers with adequate optical constants and construct the desired optical stack. Experimental curves ( $T$ and $R$ ) were simulated with SCOUT software by using the model presented in Section 2.2 for the description of the optical properties of the films. In case of oxynitrides, the Drude contribution was not used in the already described model and film thickness and the optical constants were calculated using the same parameters for the fit of $\mathrm{T}$ and $\mathrm{R}$ spectra. A good agreement between the simulated and experimental data was obtained (simulations are not shown in order do to not overload the figures) and the obtained refractive index $n$ and extinction coefficient $k$ are plotted in Fig. 3 as a function of the wavelength and for same samples shown in Fig. 2. The thicknesses of the films (Fig. 2 and Fig.3) were obtained from the fit to experimental spectral transmittance and reflectance curves, of individual layers deposited on glass are also indicated in the legend of Fig. 3. The optical constants of films deposited on copper (nitrides and oxynitrides) are found to be slightly different which is mainly due to a biased voltage.

From Fig. 3a it is seen that for nitride films the refractive index and extinction coefficient increases with the wavelength, which is a characteristic behavior of metallic films. It is also seen that the increase of nitrogen flow induces a decrease of both refractive index and

\section{a)}

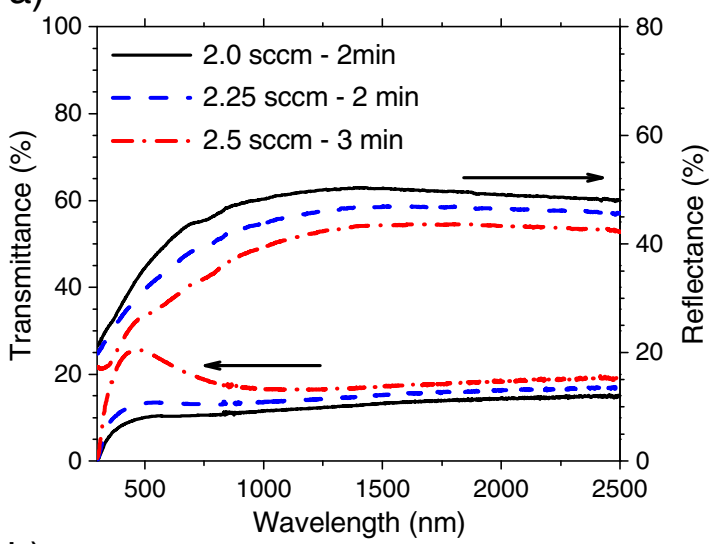

b)

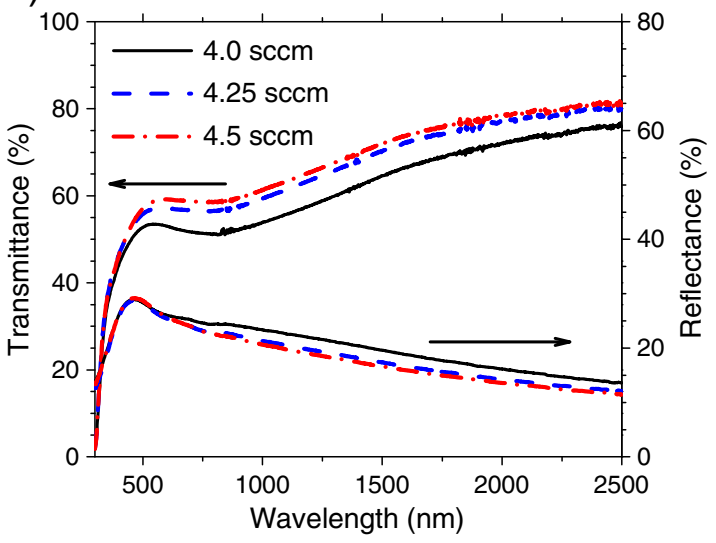

Fig. 2. Transmittance and reflectance of: a) nitrides (TiAlN) prepared with different flows of nitrogen (deposition time indicated in the legend b) oxynitrides (TiAlNxOy) prepared with different flow of gas mixture (deposition time of $2.5 \mathrm{~min}$ ). a)

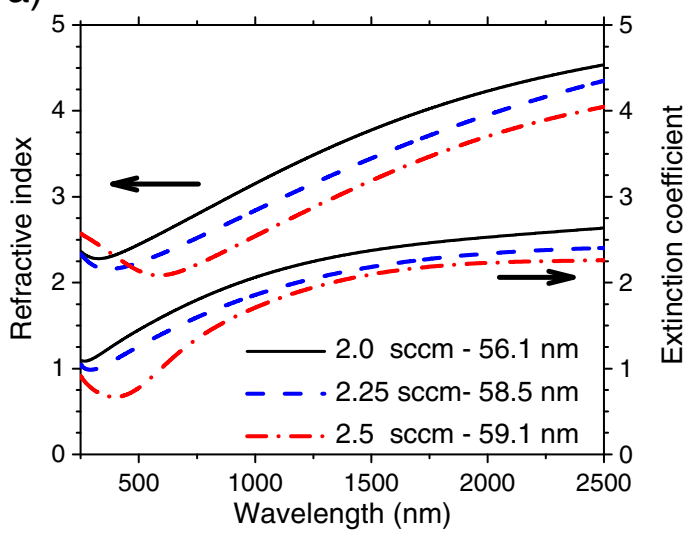

b)

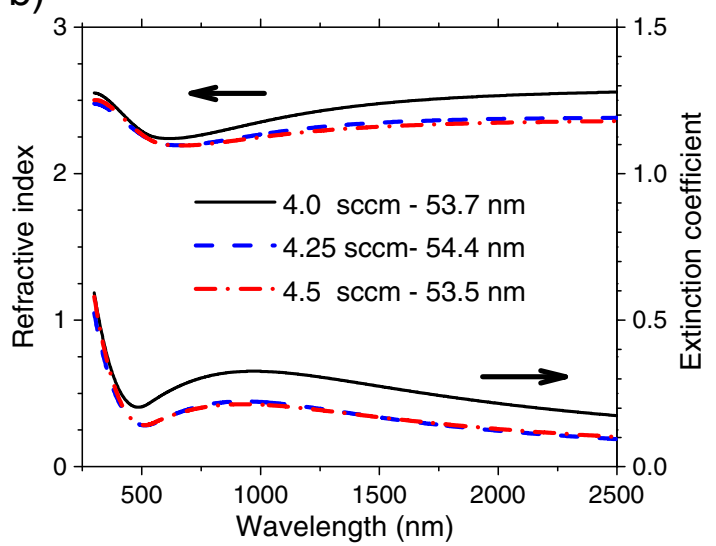

Fig. 3. Refractive index (n) and extinction coefficient ( $\kappa$ ) as a function of the wavelength of: a) nitrides (TiAlN) prepared with different flows of nitrogen; and b) oxynitrides (TiAlNxOy) prepared with different flow of gas mixture. The thicknesses calculated with the fit are also indicated in the legend.

extinction coefficient. The deposition rate also revealed a substantial decrease, from $\sim 28 \mathrm{~nm} / \mathrm{min}$ to $\sim 19.7 \mathrm{~nm} / \mathrm{min}$, when the nitrogen flow rate varied from $2 \mathrm{sccm}$ to $2.5 \mathrm{sccm}$, which shows a transition from metallic mode to reactive mode deposition.

In the case of oxynitrides (Fig. 3b), the refractive index does not show appreciable variation with wavelength and the extinction coefficient reveals a maximum around $1000 \mathrm{~nm}$ and then it decreases, which is a expected behaviour for a semiconductor material. Again, a variation of gas mixture flow is reflected in a variation of the optical

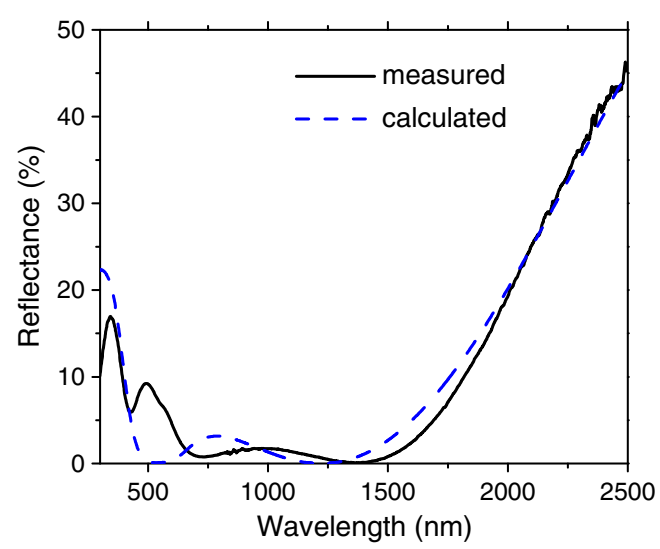

Fig. 4. Calculated and measured reflectance of TiAlN/TiAlNxOy/SiO ${ }_{2}$ tandem absorber deposited on copper. 


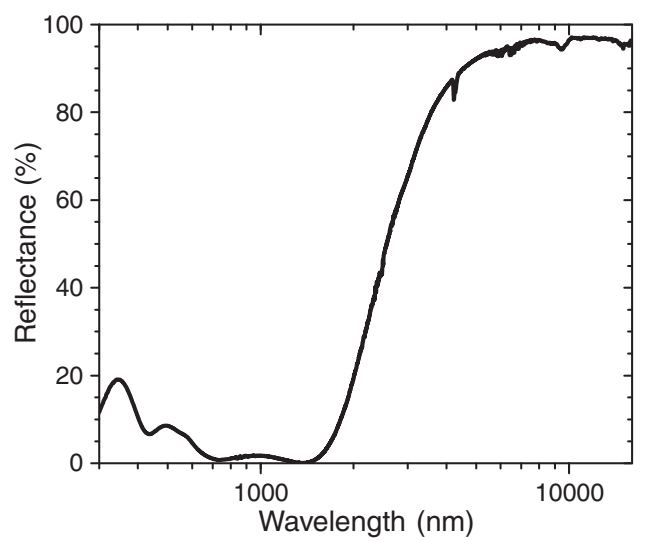

Fig. 5. Reflectance of tandem absorber deposited on copper measured with a UV/Vis/NIR (from 0.25 to $2.5 \mu \mathrm{m}$ ) and a FTIR (wavelength range $2.5-16 \mu \mathrm{m}$ ) spectrophotometers.

constants (refractive index and extinction coefficient). As expected, the extinction coefficient of $\mathrm{SiO}_{2}$ is almost zero, which is consistent with a dielectric behaviour and the refractive index is below than 1.5 (not shown due to space limitations), which makes this material a good antireflection layer.

In order to build the 3 layers TiAlN/TiAlNO/SiO 2 absorber on a copper substrate, several simulations were performed using the optical properties of the substrate and of different nitrides and oxynitrides layers. The calculated reflectance for the best layer combination (solar absorptance of 96.6\%) is shown in Fig.4, together with the measured reflectance of the corresponding produced sample (solar absorptance of $95.5 \%$ ). The layers parameters used for this absorber are: TiAlN layer ( $\mathrm{N}_{2}$ flow of $2.5 \mathrm{sccm}$ and $65 \mathrm{~nm}$ ), a TiAlN $\mathrm{O}_{\mathrm{y}}$ layer (gas mixture flow of $4.25 \mathrm{sccm}$ and $51 \mathrm{~nm}$ ), and a $\mathrm{SiO}_{2}$ layer $(100 \mathrm{~nm})$.

The thermal emittance was calculated from the spectral reflectance measured with a FTIR spectrophotometer equipped with an integrating sphere, which was used to cover the infrared wavelength range between 2.5 and $16 \mu \mathrm{m}$. The reflectance in the visible and infrared region measured at room temperature is shown in Fig. 5. The emittance was calculated for an absorber temperature of $100{ }^{\circ} \mathrm{C}$ and the tandem absorber $\mathrm{Cu} / \mathrm{TiAlN} / \mathrm{TiAlON} / \mathrm{SiO}_{2}$ presents a normal emittance of $5 \%$. The emittance of a similar sample was measured with the emissometer and revealed an emittance of $8 \%$.

Accordingly the principles defined in ref [14] a sample with a normal solar absorptance of $95 \%$ and normal thermal emittance of $5 \%$, will have a stagnation temperature of $204{ }^{\circ} \mathrm{C}$. Thus, the degradation due to thermal load (oxidation) should be tested by annealing at $278^{\circ} \mathrm{C}$ in air [14]. Tested samples were assessed after 150, 300 and $600 \mathrm{~h}$. After the thermal annealing for $600 \mathrm{~h}$ at $278{ }^{\circ} \mathrm{C}$, the solar absorptance revealed a decrease of $0.4 \%$ and the thermal emittance increased $1 \%$. The number of emittance measurements was not enough to have a good statistics, although a similar solar absorptance variation was found for several samples.

\section{Conclusions}

The paper presents the optimization process of a TiAlN/TiAlNxOy/SiO ${ }_{2}$ stack absorber deposited on copper for solar selective applications. The first two layers were prepared by magnetron sputtering while the $\mathrm{SiO}_{2}$ layer was deposited by PECVD. A theoretical analysis of transmittance and reflectance of the individual layers was used to obtain the optical constants. The dielectric function and the thickness of the different layers were calculated through the modelling of the experimental transmittance and reflectance curves by a commercial optical simulation program (SCOUT). The dielectric function of TiAlN, TiAlNxOy and $\mathrm{SiO}_{2}$ layers was considered as a contribution of intraband and interband transitions. The dielectric function was treated as the sum of Drude free carrier absorption, a number of bound harmonic oscillators, a OJL term and a background contribution. The optical constants of the individual layers were then used to construct the three layers stack minimizing the reflectance in the wavelength range below $2 \mu \mathrm{m}$ and maximizing the reflectance for wavelengths higher than $2 \mu \mathrm{m}$. With this procedure, it was obtained a stack absorber with a normal solar absorptance of $95.5 \%$ and thermal emittance of $5 \%$.

The absorber accelerated life-time tests revealed a very good oxidation resistance considering an application as in single glazed, flat-plate collectors for domestic hot water systems.

\section{References}

[1] G.A. Niklasson, C.G. Granqvist, J. Mat. Sci. 18 (1983) 3475.

[2] W. Graf, F. Brucker, M. Kohl, T. Troscher, V. Wittwer, L. Herlitze, J. Non-Cryst. Solids 218 (1997) 380.

[3] M. Farooq, Z.H. Lee, Renewable Energy 28 (2003) 1421.

[4] A. Biswas, D. Bhattacharyya, H.C. Barshilia, N. Selvakumar, K.S. Rajam, Appl. Surf. Sci. 254 (2008) 1694.

[5] Du Miao, Hao Lei, Mi Jing, Lv Fang, X. Liu, L. Jiang, S. Wang, Sol. Energy Mater. Sol. Cells 95 (2011) 1193.

[6] M. Adsten, R. Joerger, K. Järrendahl, E. Wäckelgård, Solar Energy 68 (2000) 325.

[7] V. Teixeira, E. Sousa, M.F. Costa, C. Nunes, L. Rosa, M.J. Carvalho, M. Collares-Pereira, E. Roman, J. Gago, Thin Solid Films 392 (2001) 320.

[8] Q.-C. Zhang, D.R. Mills, J. Appl. Phys. 72 (1992) 3013.

[9] R. Blickensderfer, D.K. Deardoff, R.L. Lincoln, Solar Energy 19 (1977) 429.

[10] L. Rebouta, F. Vaz, M. Andritschky, M.F. da Silva, Surf. Coat. Technol. $76-77$ (1995) 70.

[11] M. Lazarov, P. Raths, H. Metzger, W. Spirkl, J. Appl. Phys. 77 (1995) 2133.

[12] S. Venkataraj, D. Severin, S.H. Mohamed, J. Ngaruiya, O. Kappertz, M. Wuttig, Thin Solid Films 502 (2006) 228.

[13] W. Theiss, in: M. Theiss (Ed.), SCOUT Thin Film Analysis Software Handbook, Hard-and Software, Aachen, Germany (http://www.mtheiss.com).

[14] M. Kohl, M. Heck, S. Brunold, U. Frei, B. Carlsson, Sol. Energy Mater. Sol. Cells 84 (2004) 275.

[15] J.A. Duffie, W.A. Beckman, Solar Engineering of Thermal Processes, WileyInterscience, New York, 1991.

[16] T.C. Paulick, Appl. Opt. 25 (1986) 562.

[17] M. Fox, Optical properties of solids, Oxford University Press Inc, New York, 2001.

[18] S.K. O_Leary, S.R. Johnson, P.K. Lim, J. Appl. Phys. 82 (1997) 3334.

[19] C.C. Kim, J.W. Garland, H. Abad, P.M. Raccah, Phys. Rev. B 45 (1992) 11749.

[20] H. Weis, T. Muggenburg, P. Grosse, L. Herlitze, I. Friedrich, M. Wuttig, Thin Solid Films 351 (1999) 184.

[21] S. Venkataraj, J. Geurts, H. Weis, O. Kappertz, W.K. Njoroge, R. Jayavel, M. Wuttig J. Vac. Sci. Technol., A 19 (2001) 2870.

[22] P. Carvalho, F. Vaz, L. Rebouta, L. Cunha, C.J. Tavares, C. Moura, E. Alves, A. Cavaleiro, Goudeau Ph., E. Le Bourhis, J.P. Rivière, J.F. Pierson, O. Banakh, J. Appl. Phys. 98 (2005) 023715. 